\title{
Preparation and characterization of polypyrrole/graphene nanocomposite films and their electrochemical performance
}

\begin{abstract}
A one-step electrochemical process had been employed to synthesize nanocomposite films of polypyrrole/graphene (PPy/GR) by electrochemical polymerisation on indium tin oxide (ITO) from an aqueous solution containing pyrrole monomer, graphene oxide (GO) nanosheets and sodium p-toluenesulfonate (NapTS). The X-ray diffraction (XRD) patterns showed that the typical peak of GO at 9.9o was missing from the nanocomposite's diffraction pattern, suggesting that the GO had been stripped off of its oxygenous groups after the reaction. We postulated that a nanocomposite film was produced through a layer-by-layer deposition based on field emission scanning electron microscope (FESEM) images. The Raman spectroscopy profiles exhibited that the $\mathrm{D} / \mathrm{G}$ intensity ratio (ID/IG) of PPy was not altered by the inclusion of GO due to the low concentration of the material used. However, the concentration was sufficient to increase the specific capacitance of the nanocomposite by 20 times compared to that of pure PPy, reflecting a synergistic effect between PPy and GR, as analysed by a threeelectrode electrochemical cell. The electrochemical performance of the nanocomposites was affected by varying the deposition parameters such as concentrations of pyrrole and GO, scan rate, deposition time and deposition potential.
\end{abstract}

Keyword: Supercapacitor; Electrochemistry; Graphene; Polypyrrole. 